\title{
A Surface Acoustic Wave Biofilm Sensor Integrated With A Treatment Method Based On The Bioelectric Effect
}

Journal: Sensors and Actuators A

Authors: Young Wook Kim ${ }^{1,2 *}$, Mariana T. Meyer ${ }^{1,3}$, Andrew Berkovich ${ }^{2}$, Sowmya Subramanian ${ }^{1,2}$, Agis A. Iliadis ${ }^{2}$, William E. Bentley ${ }^{3}$, Reza Ghodssi ${ }^{1,2,3^{*}}$

Affiliations:

${ }^{1}$ MEMS Sensors and Actuators Laboratory, Institute for Systems Research

${ }^{2}$ Department of Electrical and Computer Engineering

${ }^{3}$ The Fischell Department of Bioengineering

University of Maryland, College Park, Maryland, USA

Corresponding Authors:

Dr. Young Wook Kim

yw78.kim@samsung.com,

150, Maeyoung-ro, Yeongtong-gu, Suwon

Gyeonggi-do 443-743, Korea

$+82-31-8093-8557$

Dr. Reza Ghodssi

ghodssi@umd.edu

2173 A.V. Williams Building

College Park, MD, USA

$+1-301-405-8158$

\begin{abstract}
Bacterial biofilms have an extensive impact on quality of life, ranging from severe infections in the clinical field to water facility contamination in environmental science. Biofilms are comprised of diverse bacteria that produce an extracellular matrix which prevents drug diffusion through them. Hence traditional antibiotic therapies require 500 - 5000 times the concentration used to eliminate non-biofilmassociated infections. Early biofilm detection is critical for effective eradication. Moreover, developing an alternative biofilm treatment method that utilizes low doses of antibiotics is desired. In this paper, a realtime microsystem is shown to detect growth of biofilms as well as their removal through integrated treatment. Detection of biofilms is achieved using a surface acoustic wave (SAW) sensor that monitors the total biomass by measuring the resonant frequency of the system. Biofilm treatment is based on the bioelectric effect (BE), a combination of low-dose antibiotics with application of both alternating and direct current signals. The detection limit of the SAW system is approximately $166 \mathrm{pg}$, corresponding to a bacterial population on the order of hundreds of bacteria. The system is used to observe an $80 \%$ reduction of total biomass when treated by the BE as compared to traditional antibiotics. Through system integration of the BE with the SAW sensor, simultaneous biofilm detection and treatment is achieved. The system consumes $194 \mu \mathrm{W}$ of power, with the sensor and treatment consuming $100 \mu \mathrm{W}$ and $94 \mu \mathrm{W}$, respectively. The integrated sensing and treatment capabilities of this system advance the development of an innovative biofilm control method.
\end{abstract}

Keywords: Biofilms, Surface Acoustic Wave, Bioelectric Effect, Microfluidics 


\section{Introduction}

Biofilms are complex communities composed of a population of bacteria surrounded by an extracellular matrix $^{1}$. Bacteria in biofilms readily exchange genetic material ${ }^{2}$, creating a heterogeneous bacterial composition more likely to be resistant to antimicrobials than a bacterial population in suspension ${ }^{3,4}$. The extracellular matrix is composed of diverse polysaccharides and proteins, among other biological molecules, such as DNA fragments ${ }^{5}$. The matrix maintains the mechanical structure of the biofilm. The physiological function of the matrix is to act as a protective layer against diffusion of antibiotics and prevent recognition by the host immune system ${ }^{6}$. Once biofilms are established, elimination of the biofilm usually requires $500-5000$ times the antibiotic inhibitory dose used on suspended bacteria ${ }^{7}$. Hence, detection during the onset of biofilm formation and during the growth stages of the biofilm, before mechanical and physiological structures have completely formed, enables its treatment using low doses of antibiotics $^{8}$. Continuous monitoring of biofilm growth is critical for detecting the onset of biofilm formation ${ }^{9}$. More importantly, developing a new alternative biofilm treatment method that utilizes low doses of antibiotics is required to prevent the formation of mature biofilm infections.

Microfluidics based systems can be ideal platforms for biofilm applications since they require small sample volume, typically nanoliters, and provide precisely controlled conditions including uniform nutrient and electrolyte distribution. Microfluidic systems have been widely utilized in biofilm research to investigate the efficacy of new drugs ${ }^{10}$ and also to monitor biofilm growth in real time ${ }^{11}$.

In this paper we integrate a microfabricated surface acoustic wave (SAW) ${ }^{12}$ sensor for real time biofilm growth monitoring with electric field enhanced treatment of mature biofilms. Previously, a microfluidic platform based on optical detection was used for the real time study of this biofilm inhibition method, showing improved treatment efficacy based on the bioelectric effect $(\mathrm{BE})^{13}$. The platform was composed of microfluidic flow reactors for biofilm growth and electrodes patterned along the channel to provide electric fields for the BE. The detection of biofilms was performed by measuring the optical density (OD) using photodiodes. For the biofilm treatment, an electric signal with increased total electrical energy, 0.25 
$\mathrm{V}$ amplitude sinusoidal signal at $10 \mathrm{MHz}$ with a $0.25 \mathrm{~V} \mathrm{DC}$ offset, was applied in combination with low doses of the antibiotic gentamicin $(10 \mu \mathrm{g} / \mathrm{mL})$ for the BE. The voltage applied to induce the electric field was maintained at less than $0.82 \mathrm{~V}(0.25 \mathrm{~V}$ for $2 \mathrm{~mm}$ spaced electrodes or $1.25 \mathrm{~V} / \mathrm{cm}$ of electric field $)$ to avoid electrolysis of the surrounding medium ${ }^{14}$ and the frequency of the AC component was selected to be $10 \mathrm{MHz}$ based on literature ${ }^{15}$. The antibiotic concentration $(10 \mu \mathrm{g} / \mathrm{mL}$ of gentamicin) utilized for the BE was significantly lower than the concentration of antibiotic typically necessary for biofilm treatment $(\sim 500-5000 \text { times higher than planktonic Escherichia coli, MIC, gentamicin } \approx 2-4 \mu \mathrm{g} / \mathrm{mL})^{16}$. Compared to only antibiotic treatment $(10 \mu \mathrm{g} / \mathrm{mL}$ of gentamicin), the BE applied to biofilms within the microfluidic device reduced biofilms by about $160 \%$. However, since the photodiodes and the optical source are required to be positioned out-of-plane from the biofilms, systems utilizing optical elements are inherently difficult to integrate into biomedical implants. In order to overcome this shortcoming, an inplane method for total biofilm detection, such as a piezoelectric sensor is required, that not only detects biofilm growth in real time but can also be used to apply the BE based treatment method in clinical applications including portable biomedical devices. Piezoelectric sensors also provide the advantage of higher sensitivity to change in biomass as compared to the optical methods.

A SAW sensor was previously developed using zinc oxide $(\mathrm{ZnO})$ based on its high piezoelectric coefficient and biocompatibility ${ }^{17}$. For reliable operation of the sensor, it was coated with an aluminum oxide $\left(\mathrm{Al}_{2} \mathrm{O}_{3}\right)$ layer deposited using atomic layer deposition (ALD). This protected the $\mathrm{ZnO}$ from degradation caused by the bacterial growth media ${ }^{18}$. As a result of the high quality passivation layer and the crystallized (c-axis) low impurities of the $\mathrm{ZnO}$ film deposited via pulsed laser deposition (PLD), the SAW sensor demonstrated reliable biofilm detection in real time. Here, the SAW sensor was integrated with a second set of electrodes for biofilm treatment based on the combination of electric field with antibiotics (bioelectric effect). The reliable operation of the SAW sensor in biological media, its high sensitivity for biofilm detection, as well as its two dimensional structure, merits to be a platform for a microsystem for biofilm applications. As discussed below, an integrated device combining the SAW 
sensor with an additional pair of electrodes was fabricated and tested by establishing and treating biofilms on the system. E. coli K-12 W3110 and Pseudomonas aeruginosa PAO1 biofilms were chosen as models of clinically relevant biofilms ${ }^{19}$. The device demonstrated successful real-time monitoring of biofilms as well as significantly improved treatment of the biofilms formed by both strains of bacteria.

\section{Figure 1}

\section{Material and Method}

The microsystem, shown in Figure 1, consists of the biofilm detection component using the SAW sensor and a biofilm treatment component using an electrical signal combined with antibiotics. To ensure seamless integration of the sensor with the treatment electrodes, theoretical analyses were conducted for two important factors that could significantly impact the sensitivity of the SAW sensor: 1) interference due to the application of the BE electric field, and 2) effect of shear stress due to the microfluidic flow. Since the acoustic wave is induced by the electrical actuation of the piezoelectric material, the integration of the BE electric field for biofilm treatment can attenuate the SAW propagation, due to the proximity of the BE field ${ }^{20}$. The microfluidic flow can potentially alter SAW propagation, since the flow induces surface stress $^{21}$ that may alter SAW velocity, resulting in a decrease in sensitivity.

\section{Figure 2}

\subsection{Analysis of electric field interference due to the BE}

To assess the first mode of potential interference, studies of the electrical signal interference between the BE electric field and the acoustic waves were conducted using the schematic of the device shown in Figure 2a. The $\mathrm{Al}_{2} \mathrm{O}_{3}$ film deposited on the $\mathrm{ZnO}$ layer was assumed to be separated by an air gap on each side from the treatment electrodes. This assumption was made to investigate the maximum electric field that can be induced in this configuration. Since the fabricated devices were tested in bacterial growth media or deionized (DI) water, the actual voltage drop between the electrode and the $\mathrm{Al}_{2} \mathrm{O}_{3}-\mathrm{ZnO}$ layer 
(from 0 to $\mathrm{X}_{1}$ in Figure 1) is smaller than the case of air gap due to the higher relative dielectric constant of water as compared to air $\left(\varepsilon_{\text {r-air }}=1 . \varepsilon_{\text {r-water }}=80.1\right.$ at $\left.20^{\circ} \mathrm{C}\right)$.

The induced field intensity on the $\mathrm{ZnO}$ layer due to the $\mathrm{BE}$ voltage applied $(0.25 \mathrm{~V}$ DC superimposed on a $0.25 \mathrm{~V}$ sinusoidal signal at $10 \mathrm{MHz}$ ) was calculated based on electromagnetic theory (Maxwell's equation, Gauss's law ${ }^{22}$ as:

$$
\begin{aligned}
& E=\frac{Q}{2 A \varepsilon_{Z n O}} \approx \frac{C_{\text {total }} V_{0}}{2 A\left(100 \cdot \varepsilon_{0}\right)}=\left(\frac{\varepsilon_{\text {total }} A}{d}\right) \frac{V_{0}}{200 A \varepsilon_{0}}=\left(\frac{\varepsilon_{\text {total }}}{d}\right) \frac{V_{0}}{200 \varepsilon_{0}} \\
& \frac{d}{\varepsilon_{\text {total }}}=\frac{2 w_{\text {air }}}{\varepsilon_{0}}+\frac{2 w_{A l_{2} O_{3}}}{9.8 \varepsilon_{0}}+\frac{w_{Z n O}}{100 \varepsilon_{0}}=\frac{10.1 \times 10^{-4}[\mathrm{~m}]}{\varepsilon_{0}} \\
& E \approx\left(\frac{\varepsilon_{\text {total }}}{d}\right) \frac{V_{0}}{200 \varepsilon_{0}}=\frac{V_{0}}{200 \varepsilon_{0}} \cdot\left(\frac{\varepsilon_{0}}{10.1 \times 10^{-4}[\mathrm{~m}]}\right)=1.238[\mathrm{~V} / \mathrm{m}]
\end{aligned}
$$

where $Q$ is the induced charge due to the applied voltage, $A$ is area of the side electrode, $\varepsilon_{Z n O}$ is dielectric constant of $\mathrm{ZnO}\left(\sim 100 \varepsilon_{0}\right), d$ is distance between the electrodes $(2 \mathrm{~mm}), W_{A l 2 O 3}$ is the thickness of $\mathrm{Al}_{2} \mathrm{O}_{3}$ $(100 \mathrm{~nm}), V_{0}$ is the magnitude of the applied voltage $(0.25 \mathrm{~V}), W_{\text {air }}$ is width of air gap $(0.3999 \mathrm{~mm}), W_{Z n O}$ is width of the $\mathrm{ZnO}(1.2 \mathrm{~mm}), \varepsilon_{0}$ is the vacuum permittivity, $\varepsilon_{\text {total }}$ is the total dielectric constant that can be calculated by the electrical capacitance of the system. The relative dielectric constant of each layer indicates the characteristics of the polarity of the medium and directly corresponds to the induced electric field intensity. With device design parameters, the intensity of the electric field was calculated to be 1.238 $\mathrm{V} / \mathrm{m}$.

Based on the obtained value, the induced displacement of the $\mathrm{ZnO}$ was derived using the general compressed tensor equation $^{23}$ and a $\mathrm{ZnO}$ piezoelectric coefficient obtained from literature ${ }^{24}$.

$$
S=d \cdot E
$$


where $S$ is the strain due to the applied electric field $(E=1.238 \mathrm{~V} / \mathrm{m}), d$ is piezoelectric strain coefficient $\left(12.4 \times 10^{-12} \mathrm{C} / \mathrm{N}\right)$. The result of displacement due to the electric effect is approximately $15.4 \times 10^{-15} \mathrm{~m}$. The wavelength of the SAWs on the sensor was previously characterized by our group using the phase velocity of $\mathrm{ZnO}(4812 \mathrm{~m} / \mathrm{s})$ and the distance between the electrodes (interdigitated transducer, $6 \mu \mathrm{m}$

distance). This design generated a $402 \mathrm{MHz}$ acoustic wave on the $\mathrm{ZnO}^{12}$. The resonant frequency is designed based on the suggested frequency range (401 - $406 \mathrm{MHz}$, Federal Communication Commission) for biomedical wireless application. The induced attenuation of the SAW due to the application of the BE electric field is nine orders of magnitude smaller than the wavelength of the acoustic waves (12 $\mu \mathrm{m})$. Therefore, it was concluded that possible electrical interferences between propagation of the SAWs and the electric field for the BE in an integrated scheme were not significant.

\subsection{Analysis of shear stress on the SAWs}

The impact of the shear stress imposed by the microfluidic flow on SAW propagation was investigated as the second factor with potential to reduce performance. Conditions of laminar flow ${ }^{25}$ were assumed for this analysis, as the Reynolds number ${ }^{26}$ of the microchannel $(100 \mu \mathrm{m}$ height, $2 \mathrm{~mm}$ width, $2 \mathrm{~cm}$ length and $20 \mu \mathrm{L} /$ hour $=28 \mu \mathrm{m} / \mathrm{s}$ in the channel) and dynamic viscosity of water $(0.001 \mathrm{~Pa} \cdot \mathrm{s})$ is calculated to be $\sim 0.056$ which is less than the 2100 laminar flow threshold ${ }^{27}$. A schematic illustrating the laminar flow condition that was used in this analysis is shown in Figure 2b. In order to investigate the change in the acoustic wave velocity purely due to the microfluidic flow, and not due to the passivation layer that adds to the dampening force, it was also assumed that the shear stress is applied to a non-passivated $\mathrm{ZnO}$ film. The shear stress on the substrate is known to be proportional to the velocity profile of the laminar flow. The derived velocity slope and shear stress $(\tau)$ due to the velocity profile are shown in equation 3 and 4 , respectively ${ }^{28}$.

$$
\left.\frac{\partial u}{\partial y}\right|_{y=0}=\left.\frac{\Delta u}{\Delta y}\right|_{y=0}=\frac{2 u_{0}}{h}
$$




$$
\tau_{0}=\left.\mu \frac{\partial u}{\partial y}\right|_{y=0}=\mu \frac{2 u_{0}}{h}
$$

where $u_{0}$ is the maximum velocity of the flow, $h$ is the height of the channel, $\mu$ is the dynamic viscosity of the fluid and $\tau_{0}$ is the shear stress at the bottom of the channel (figure 2b). The shear stress on the SAWs can alternatively be derived from the applied force per unit area. Based on the known relation of acceleration and velocity, the surface tension can be expressed as shown in equation 5 .

$$
\tau_{0}=\frac{F}{A}=\frac{1}{A}\left(\frac{m_{Z n O} \Delta v}{\Delta t}\right)=\frac{1}{A}\left(\frac{m_{Z n O} \Delta v}{\Delta t}\right) \cdot \frac{d_{Z n O}}{d_{Z n O}}=\left(\frac{\rho_{Z n O} \Delta v}{\Delta t}\right) d_{Z n O}
$$

where $F$ is applied force, $A$ is area where the force is applied, $m_{Z n O}$ is the mass of the $\mathrm{ZnO}, \Delta v$ is the change in SAW velocity at given time $(\Delta t)$ due to the applied force, $\rho_{Z n O}$ is the density of the $\mathrm{ZnO}$ and $d_{Z n O}$ is the thickness of the $\mathrm{ZnO}$ layer. $\Delta t$ was calculated as the time taken for the SAWs to propagate from the input interdigitated electrode (IDT) to output IDT of the sensor. The speed of the SAW on the $\mathrm{ZnO}$ layer $(4812 \mathrm{~m} / \mathrm{s})$ is an intrinsic material property ${ }^{14}$, and the distance between the two IDTs (100ג) were characterized previously ${ }^{13}$. Combining equations 4 and 5 , the change in the SAW velocity $(\Delta v)$ due to the laminar flow is given by equation 6.

$$
\Delta v=\mu \frac{2 u_{0}}{h} \cdot \frac{100 \lambda}{u_{s}} \cdot \frac{1}{\rho_{Z n O} \cdot d_{Z n O}}
$$

Based on the laminar flow velocity profile equation ${ }^{25}$, the maximum flow rate $\left(\mathrm{v}_{\mathrm{m}}\right)$ is 1.5 times of the average flow velocity $\left(\mathrm{v}_{\text {avg }}\right)$ as shown below.

$$
\begin{aligned}
& v_{\text {avg }}=\frac{1}{R} \int_{0}^{R} v_{m}\left(1-\frac{r^{2}}{R^{2}}\right) d r=\frac{1}{R} \cdot \frac{2}{3} R v_{m} \\
& \therefore v_{m}=\frac{3}{2} v_{\text {avg }}
\end{aligned}
$$


Using relevant values including maximum flow speed (1.5 times of the average velocity, $1.5 \times 28=42$ $\mu \mathrm{m} / \mathrm{s}), \mathrm{ZnO}$ density $\left(5.61 \mathrm{~g} / \mathrm{cm}^{3}\right)$, thickness of the $\mathrm{ZnO}(500 \mathrm{~nm})$, viscosity of water $\left(1.002 \times 10^{-3} \mathrm{~N} \cdot \mathrm{S} / \mathrm{m}^{2}\right)$, wavelength of the SAW $(12 \mu \mathrm{m})$ and height of the channel $(100 \mu \mathrm{m})$, the velocity change of the SAW $(\Delta v)$ due to the microfluidic flow was calculated as $1.5 \times 10^{-4} \mathrm{~m} / \mathrm{s}$. This speed attenuation corresponds to a 12.5 $\mathrm{Hz}$ shift in frequency as shown in below.

$$
f=\frac{\Delta v}{\lambda}=\frac{1.5 \times 10^{-4}[\mathrm{~m} / \mathrm{s}]}{12 \times 10^{-6}[\mathrm{~m}]}=12.5 \mathrm{~Hz}
$$

Since typical resonant frequency changes of the SAW sensor for detecting biofilms grown on its surface are approximately $1 \mathrm{MHz}^{13}$, the effect of microfluidic flow on the SAW is five orders of magnitude smaller than the signal of the sensor. Considering the actual device includes an $\mathrm{Al}_{2} \mathrm{O}_{3}$ passivation film over the $\mathrm{ZnO}$, which avoids direct contact of the fluid and the piezoelectric layer, the resonant frequency attenuation is expected to be lower than the $12.5 \mathrm{~Hz}$. This is because the ALD passivation film absorbs the shear stress prior to propagation to the $\mathrm{ZnO}$ layer.

\section{Figure 3}

\subsection{Fabrication}

The fabrication process flow for the device is shown in Figure 4. The fabrication of the SAW sensor component has been previously described ${ }^{13}$. The process includes fabrication of Au IDT electrodes with 2 $\mu \mathrm{m}$ width and spacing that are patterned using lift-off. A ZnO piezoelectric film with a thickness of 500 $\mathrm{nm}$ was deposited using pulsed laser deposition. PLD was selected due to its ability to deposit high quality single crystal piezoelectric films with extremely low concentrations of impurities. The orientation of the $\mathrm{ZnO}$ is well controlled due to the high stochastic correlation with the crystal structure of the target material ${ }^{29}$. After $\mathrm{ZnO}$ deposition and annealing, the sensor was passivated with $110 \mathrm{~nm} \mathrm{Al}_{2} \mathrm{O}_{3}$ deposited 
using atomic layer deposition (ALD) at $150{ }^{\circ} \mathrm{C}$, to protect the surface from bacterial growth media. ALD enables pin-hole free conformal films with small thicknesses and is very suitable for this application.

Polydimethylsiloxane (PDMS) microchannels to provide a constant supply of fresh bacterial growth media and the antibiotic treatment for biofilms were assembled onto the fabricated chips. PDMS provides advantages for biofilm research, such as permeability, cost-effectiveness, and ease of fabrication ${ }^{30}$. A mold of the microfluidic structures was fabricated by patterning $100 \mu \mathrm{m}$-thick KMPR-1050 on a silicon substrate using contact photolithography. PDMS (Sylgard 184, Dow Corning) in the ratio of 10:1 (base : curing agent) was degassed, poured over the mold, and cured in a furnace for 20 minutes at $80^{\circ} \mathrm{C}^{16}$. After cooling, the PDMS was removed from the mold and diced to fit the fabricated chips. Reversible bonding between the PDMS channel and the chip was achieved by soaking the PDMS in methanol for 1 minute and placing over the chip. As the methanol evaporated, the PDMS adhered onto the chip due to Van der Waals forces between the substrate and PDMS.

\section{Figure 4}

\section{Experimental Procedures and Testing Setup}

Bacterial suspensions of Escherichia coli K-12 W3110 ${ }^{31}$ and Pseudomonas aeruginosa PAO1 ${ }^{11}$ were prepared from stocks stored at $-85^{\circ} \mathrm{C}$. A frozen sample of bacteria was transferred into $5 \mathrm{ml}$ of Luria Broth (LB) media in a cell culture tube. Then, the liquid suspension was cultured in a shaker-incubator at $250 \mathrm{rpm}$ and $37^{\circ} \mathrm{C}$ for 16 hours. Following this, LB media was used to dilute the suspension to a final $\mathrm{OD}_{600} \approx 0.21-0.23$.

Biofilm growth experiments were initiated by disinfecting the microchannel of the device using $70 \%$ ethyl alcohol. The channel was then washed by flowing deionized (DI) water and LB media sequentially via a syringe pump (Cole Parmer 74900). Bacterial suspensions, prepared as above, were subsequently injected and inoculated without flow for 2 hours to allow for bacterial attachment to the substrate ${ }^{16}$. LB media was then continuously supplied to the channel for 24 hours at $20 \mu \mathrm{L} / \mathrm{h}$. The flow rate chosen has 
been previously characterized by our group and confirmed to supply sufficient nutrients for biofilm growth $^{11}$. The biofilm growth was performed for 24 hours. Continuous LB media was supplied at a flow rate of $20 \mathrm{ul} / \mathrm{hr}$ that resulted in mature biofilm formation ${ }^{11-13}$. After growth, four different treatments were applied to the biofilms for an additional 24 hours. For the antibiotic and bioelectric treatments, gentamicin - a traditional antibiotic used in the treatment of $E$. coli and $P$. aeruginosa - was added to the LB media at a concentration of $10 \mu \mathrm{g} / \mathrm{mL}$. The electric field was applied using a signal generator (Agilent 33220A) for the $\mathrm{BE}$ and for electric field-only treatment control.

After the experiments were completed, biofilms in the microfluidic channel were stained using live/dead fluorescent dyes (Syto9 / Propidium iodide, Life Technologies Inc., USA). Using a fluorescent microscope (Olympus BX60), biofilm images were obtained. The optical coverage of the images was evaluated using an image processing program (Image J 1.44, USA) that converts the images to binary with respect to the background.

Since biofilms can exhibit variable growth caused by high sensitivity to environmental conditions, performing parallel experiments to ensure similar experimental conditions across all experiments is critical to obtain results. To conduct four experiments in parallel with a single channel network analyzer (HP8510B), a custom electronic circuit board was designed using a multiplexer (MC74HC4052N, Motorola Inc.) that can be switched by a 4-bit counter (DM74LS161A, Fairchild Semiconductor Inc.). Using this setup, four experiments (i.e. control (LB media only), only antibiotic, only electric field, and antibiotic with electric field (BE)) were performed simultaneously, and the resonant frequency of each device was measured every 16 minutes.

\section{Results and Discussion}

Prior to testing device performance with bacterial biofilms, the detection limit of the system was determined by loading a known mass onto the sensor. The microfluidic channel was filled with DI water and the mass loaded on the sensing area ${ }^{13}$, between the two IDTs, as shown in Figure $4 \mathrm{~g}$, was calculated 
to be $207 \mu \mathrm{g}$ using the density of water $\left(1000 \mathrm{~kg} / \mathrm{m}^{3}\right)$. A resonant frequency change of $125 \mathrm{KHz}$ was recorded as a result of this mass loading. The linear mass sensitivity $[\mathrm{Hz} / \mathrm{g}]$ was calculated as the ratio of the observed frequency shift $(125 \mathrm{KHz})$ to the applied mass $(207 \mu \mathrm{g})$. The detection limit (resolution/sensitivity) was obtained by dividing the resolution of the network analyzer $(0.1 \mathrm{~Hz}$ for the HP8510B) with the calculated linear mass sensitivity $\left(6.04 \times 10^{8}[\mathrm{~Hz} / \mathrm{g}]\right)$.

Linear sensitivity $=\frac{\Delta \mathrm{Fr}}{\Delta \mathrm{m}}=\frac{125 \mathrm{KHz}}{207 \mu \mathrm{g}}=6.04 \times 10^{8}\left[\frac{\mathrm{Hz}}{\mathrm{g}}\right]$
Detection limit $=\frac{\text { resolution }}{\text { linear sensitivity }}=\frac{0.1[\mathrm{~Hz}]}{6.04 \times 10^{8}[\mathrm{~Hz} / \mathrm{g}]}=166 \mathrm{pg}$

The detection limit of the device is presented in table 1."

\section{Table 1}

The detection limit of $166 \mathrm{pg}$, corresponds to hundreds of bacteria based on the reported mass of a single bacterium $(\sim 1 \mathrm{pg})^{32}$. Since saturation of the sensor due to mass loading occurs at approximately $30 \mathrm{ppb}$ of the resonant frequency of the system ${ }^{33}$ (here, $402 \mathrm{MHz}$ ), the sensor theoretically saturates at approximately $30 \times 10^{-9} \times 402 \times 10^{6}=12 \mathrm{MHz}$. Biofilm growth experiments using the SAW sensor resulted in a change in frequency of only $4 \mathrm{MHz},{ }^{13}$ less than the calculated saturation limit of the sensor. Thus, the sensor is extremely sensitive to detecting and monitoring change in biofilm growth. In the biofilm experiments presented here, all devices were fabricated with a passivation layer of $\mathrm{Al}_{2} \mathrm{O}_{3}$ of $110 \mathrm{~nm}$ thickness to protect the $\mathrm{ZnO}$ layer from the growth media and achieve an extended device lifetime. With this passivation, the device was typically reusable at least five times after biofilm cleaning.

The resonant frequency of the sensor was continuously measured when either E. coli or $P$. aeruginosa biofilms were grown on the device. Within the initial 10 hours of E. coli biofilm growth, the resonant frequency of the sensor decreased by approximately $2 \mathrm{MHz}$ (Figure 5a), and subsequently saturated, 
matching expectations based on the biofilm growth cycle ${ }^{34}$. The various treatments were applied at $t=24$ hours, namely: (1) control (no treatment), (2) only electric field (1.25 V/cm amplitude sinusoidal signal with $1.25 \mathrm{~V} / \mathrm{cm}$ DC offset), (3) only gentamicin (10 $\mu \mathrm{g} / \mathrm{mL})$ and (4) BE (electric field with gentamicin). During the treatment phase, the resonant frequency was observed to fluctuate during the BE treatment in comparison to the other methods as shown in Figure 5. Since the biofilm growth in the microfluidic channel can be non-uniform ${ }^{13}$, and the results shown are the averages of three experimental repeats with the standard deviations, we hypothesize that the fluctuations are due to the statistical variation of the repeated experiments.

After treatment, the BE showed approximately a $1.6 \mathrm{MHz}$ resonant frequency increase from the start of treatment that corresponds to significant reduction of total biomass, while the antibiotic or electric field treatment does not demonstrate any significant frequency decrease (no biofilm reduction).

In the case of $P$. aeruginosa, the biofilms were grown in the microchannel for 24 hours under the same conditions. $P$. aeruginosa biofilms growth resulted in approximately $0.75-1.2 \mathrm{MHz}$ change in frequency after 10 hours of biofilm growth, corresponding to a significant increase in biomass (approximately hundreds of milligrams or $10^{9}$ bacterial cells). After biofilm treatment using the BE, significant reduction in $P$. aeruginosa biomass was observed similar to the results obtained with the $E$. coli biofilms (Figure 5b). In $P$. aeruginosa biofilms, the $\mathrm{BE}$ treatment resulted in approximately a $0.6 \mathrm{MHz}$ frequency increase when compared to the start of the treatment at $t=24$ hours.

\section{Figure 5}

After the experiments were completed, the biofilms were stained with a fluorescent dye to visualize the viable bacteria. Representative images of live E. coli (Figure 6a-d) and P. aeruginosa (Figure 7a-d) biofilms after each treatment are shown herein. The control biofilms (control, Figure 6a and 7a) show significant amounts of live bacteria (green fluorescence, E. coli: $38.1 \%$ and P. aeruginosa: $13.6 \%$ surface coverage). The antibiotic treatment does not yield effective biofilm removal, though its effect is 
significant in the case of E. coli (bacterial surface coverage: E. coli $22.6 \%$, P. aeruginosa $13.2 \%$ ). Electric field treatment did not have any significant impact (bacterial surface coverage: E. coli 41.1\%, P. aeruginosa 12.9\%). Only BE treatment (Figure $6 \mathrm{~d}$ and $7 \mathrm{~d}$ ) demonstrates an almost complete elimination of viable cells (bacterial surface coverage: E. coli: $2.5 \%, P$. aeruginosa $1.7 \%$ ). These results correlate well with the total biomass change monitored by the SAW sensor (Figure 5).

\section{Figure 6}

\section{Figure 7}

In the E. coli and $P$. aeruginosa biofilm experiments, the antibiotic treatment ( $10 \mu \mathrm{g} / \mathrm{mL}$ of gentamicin) does not show significant biofilm reduction. Biofilm insensitivity to antibiotic treatment at these concentrations is expected, as biofilm treatment typically requires approximately one thousand times more antibiotic than the concentration used for treatment of freely suspended bacteria $(\sim 2-5 \mu \mathrm{g} / \mathrm{mL}$ of gentamicin). This insensitivity might be attributable to the extracellular matrix of the biofilm that hinders drug diffusion through $\mathrm{it}^{35}$. Additionally, treatment with only electric field in either E. coli or $P$. aeruginosa strains did not result in a decrease in biomass since the intensity of the electric signal $(0.25 \mathrm{~V})$ was lower than the threshold of electrolysis $(0.82 \mathrm{~V})$ and not sufficient to impose biocidal effects on the biofilms ${ }^{36}$. Only the BE treatment that combines electric field with antibiotics demonstrated an increased efficacy of $80 \%$ and $70 \%$ biomass reduction in E. coli and P. aeruginosa biofilms respectively, as compared to the antibiotic treatment alone.

The established hypothesis for the mechanism of action of the bioelectric effect is based on the effect of the external electric field on the bacterial cell membrane that contains a variety of partially charged molecules including cell proteins ${ }^{37}$. The various partial charges in the phospholipid bilayer membrane can be distorted by the application of an external electrostatic force. As a result the bacterial membrane is partially ruptured due to the electric field, which treatment efficacy is dramatically enhanced when the electric fields are combined with small doses of antibiotics ${ }^{38}$. Comprehensive studies with diverse strains 
of bacteria have also been conducted in literature and improved biofilm treatment has been demonstrated when electric fields were applied simultaneously with antibiotics ${ }^{39}$. Results from the system developed herein complement previously published results for both E. coli and P. aeruginosa biofilms.

Importantly, the system developed can monitor any change in total biomass in real-time without using a bulky external equipment, such as an optical source and detector that are traditionally required for biomass quantification (i.e. optical density measurement), thereby enabling studies to verify the biofilm treatment efficacy of new methods. Furthermore, this system can be used towards characterizing and optimizing parameters of existing treatments. For instance, by varying the time of application of treatment (i.e. earlier than 24 hours or later), the antibiotic dose, and intensities of the electric fields, the efficacy of the BE-based biofilm treatment can be studied and monitored in real-time. Such experiments can provide significant information about the mechanism of action of the BE, and more importantly the role and effect of the various parameters of the treatment. Finally, based on the advantage of in-plane structure of the system requiring only hundreds of micro-watt electric power for operation, this system can be further developed as a micro-scale device for biomedical applications toward biofilm management in implants.

\section{Conclusion}

An integrated microsystem was designed and used to successfully demonstrate continuous real-time biofilm detection as well as effective treatment. The sensing component of the system provides the potential for early detection of biofilm growth, where traditional treatment methods are still effective. The treatment component allows for elimination even of mature biofilms with only low-dose antibiotics via the $\mathrm{BE}$. The device was used to monitor the change in total biomass in real time for a number of treatment conditions. Both E. coli and $P$. aeruginosa biofilms were tested using this microsystem. The electric field induced by the planar electrodes, when combined with small doses of the antibiotic gentamicin, resulted in a significant reduction in total biomass as compared to treatment with antibiotic alone. These results suggest that the designed system can be used for treatment of diverse biofilms at low antibiotic 
concentrations. The system integration of sensor and treatment into a single platform demonstrated here can be used for managing biofilm associated problems. Also, this platform can be used for fundamental chemical and biological research, such as new drug discovery studies.

\section{Acknowledgements}

The authors would like to acknowledge the funding support provided by the Robert W. Deutsch Foundation. The authors also acknowledge the funding support provided by NSF (CBET \#1160005, CBET \#1264509) and DTRA (HDTRA1-13-1-00037). The authors appreciate the support of the Maryland Nanocenter and its Fablab staff.

\section{References}

${ }^{1}$ Costerton J.W., Stewart P.S., Greenberg E.S., 1999, Science, 284, 1318 - 1322.

${ }^{2}$ Stoodley P., Saue K., Davies D.G., Costerton J.W., 2002, Annu. Rev. Microbiol., 56, 187 - 209.

${ }^{3}$ Fux C. A., Stoodley P., Hall-Stoodley L., Costerton J. W., 2003, Expert Rev. Anti-infect. Ther., 1, 667 - 683.

${ }^{4}$ Darouiche R. O., 2004, N. Engl. J. Med., 1422-1429.

${ }^{5}$ Hall-Stoodley L., Costerton J. W., Stoodley P, 2004, Nature reviews. Microbiology, 2, 95-108.

${ }^{6}$ Donlan R. M., 2001, Emerging infectious diseases, 7, 277-281.

${ }^{7}$ Hall-Stoodley L., Stoodley P., 2009, Cell. Microbiol., 11(7), 1034-1043.

${ }^{8}$ Heuer W., Elter C., Demling A., Neumann A., Suerbaum S., Hannig M., Heidenblut T., Bach F. W., StieschScholz M., 2007, J. Oral. Rehabil., 34, 377-382.

${ }^{9}$ Kadurugamuwa J. L., Sin L., Albert E., Yu J., Francis K., DeBoer M., Rubin M., Bellinger-Kawahara C., Parr Jr T. R., Contag P. R., 2003, Infect. Immun., 71(2), 882-890.

${ }^{10}$ Roy V., Meyer M. T., Smith J. A. I., Gamby S., Sintim H. O., Ghodssi R., Bentley W. E., 2013, Appl. Microbiol., Biotechnol., 97, 2627-2638.

${ }^{11}$ Meyer M.T., Roy V., Bentley W.E., Ghodssi R., 2011, J. Micromech. Microeng., 21, 054023.

${ }^{12}$ Kim Y.W., Sardari S.E., Meyer M.T., Iliadis A.A., Wu H.C., Bentley W.E., Ghodssi R., 2012, Sensor. Actuat. BChem., 163, 136-145. 
${ }^{13}$ Kim Y. W., Mosteller M. P., Meyer M. T., Ben-Yoav H., Bentley W. E., Ghodssi R., 2012, A Solid-State Sensors, Actuators and Microsystems Workshop, 233-236.

${ }^{14}$ Carmo M., Fritz D.L., Mergel J., Stolten D., 2013, Int. J. Hydrogen Energ., 38, 4901-4934.

${ }^{15}$ Giladi M., Porat Y., Blatt A., Shmueli E., Wasserman Y., Kirson E.D., Palti Y., 2010, Antimicrob. Agents Chemother., 52, 2803-2809.

${ }^{16}$ Ceri H., Olson M.E., Stremick C., Read R.R., Morck D., Buret A, 1999, J. Clin. Microbiol., 37, 1771-1776.

${ }^{17}$ Krishnamoorthy S., Iliadis A.A., 2008, Solid State Electron., 52, 1710-1716.

${ }^{18}$ Kim Y.W., Sardari S.E., Iliadis A.A., Ghodssi R., 2011, $11^{\text {th }}$ International Conference on Atomic Layer Deposition, 257.

${ }^{19}$ Linsay D., von Holy D., 2006, J. Hosp. Infect., 64, 313-325.

${ }^{20}$ Krishnamoorthy S., Iliadis A.A., 2006, Solid State Electron., 50, 1113-1118.

${ }^{21}$ Lu H., Koo L.Y., Wang W.M., Lauffenburger D.A., Griffith L.G., Jensen K.F., 2004, Anal. Chem., 76, 5257-5264.

${ }^{22}$ Cheng D.K., Field and Wave Electromagnetics (Addison - Wesley, 1989) [second edition]

${ }^{23}$ Main J.A., Garcia E., Newton D.V., 1995, J. Guid. Control Dynam., 18, 1068-1073.

${ }^{24}$ Emanetoglu N.W., Gorla C., Liu Y., Liang S., Li Y., 1999, Mat. Sci. Semicon. Proc., 2, 247-252.

${ }^{25}$ Kamholz A.E., Yager P., 2001, Biophys. J., 80, 155-160.

${ }^{26}$ Purcell E.M., 1976, AIP Conf. Proc., 28, 49-64.

${ }^{27}$ Holman, J. P., 2002, Heat Transfer. McGraw-Hill. p. 207.

${ }^{28}$ Wang X., Milner T.E., Chen Z., Nelson J.S., 1997, Appl. Optics, 36, 144-149.

${ }^{29}$ Pulsed Laser Deposition of Thin Films, by Douglas B. Chrisey (Editor), Graham K. Hubler (Editor), pp. 648. ISBN 0-471-59218-8. Wiley-VCH, May 2003.

${ }^{30}$ Christensen A.M., Chang-Yen D.A., Gale B.K., 2005, J. Micromech. Microeng., 15, 928-934.

${ }^{31}$ Wang L., Li J., March J.C., Valdes J.J., Bentley W.E., 2005, J. Bacteriol., 187, 8350-8360.

${ }^{32}$ Loferer-Krobbacher M., Klima J., Psenner R., 1998, Appl. Environ. Microbiol., 64, 688-694.

${ }^{33}$ Fischerauer G., "Surface Acoustic Wave Devices," in: W.Göpel, J.Hesse, J.N.Zemel, H.Meixner, R.Jones (Eds.),Sensors. A Comprehensive Survey, Vol.8. Weinheim: VCH, 1995

${ }^{34}$ Skowlund C.T., 1990, Biotechnol. Bioeng., 35(5), 502-510.

${ }^{35}$ Douglas L.J., 2003, Trends Microbiol., 11(1), 30-36.

${ }^{36}$ Korachi M., Turan Z., Senturk K., Sahin F., Aslan N., 2009, J. Electrostat., 67, 687-685. 
${ }^{37}$ Salton M.R.J., 1967, Annu. Rev. Microbiol., 21, 417-442.

${ }^{38}$ Teorell T., 1948, Nature, 162, 961.

${ }^{39}$ Del Pozo J.L., Rouse M.S., Patel R., 2008, Int. J. Artif. Organs., 31(9), 786-795. 


\section{Figure Legend}

Figure 1: Schematic of the integrated microsystem of the SAW sensor and electrodes for induction of the bioelectric effect. A microfluidic chamber is placed over the system to introduce bacterial growth media and antibiotics.

Figure 2: (a) Schematic of the device cross-section for electric field distribution analysis. The $\mathrm{ZnO}$ can affected by the voltage applied for the bioelectric effect during the biofilm treatment. The simulation was focused on the $\mathrm{ZnO}$ layer from $\mathrm{X}_{2}$ to $\mathrm{X}_{3}$ locations. $\varepsilon$ represents a dielectric constant of the each medium. (b) Schematic of the laminar flow on the SAW. The theoretical analysis was focused on the impact of the SAW speed changes due to the flow.

Figure 3: (a) Photo of the fabricated device. (b) Optical microscopy image of the integrated device. Biofilm is localized between the electrodes for the bioelectric effect (BE) via a microfluidic channel. Biofilm sensing is based on the total biomass between the two IDTs and treatment is applied when electric signal was applied to the electrodes for the $\mathrm{BE}$ as antibiotics are supplied through the microchannel.

Figure 4: Overall schematic process flow. (a) $60 \mathrm{~nm} \mathrm{PECVD} \mathrm{SiO}_{2}$ deposition and interdigitated electrode patterning and deposition ( $\mathrm{Au}$ ) by electron beam evaporation, (b) $500 \mathrm{~nm} \mathrm{ZnO}$ deposition using pulsed laser deposition, (c) $\mathrm{ZnO}$ patterning using weak acid etchant (1:1:30 ratio of acetic acid: phosphoric acid: deionized water), (d) $100 \mathrm{~nm}$-thick atomic layer deposition of $\mathrm{Al}_{2} \mathrm{O}_{3}$ at $150{ }^{\circ} \mathrm{C}$, (e) electrode patterning and fabrication via $\mathrm{Au}$ evaporation $(\mathrm{Cr} / \mathrm{Au}, 15 \mathrm{~nm} / 200 \mathrm{~nm})$ and (f) PDMS microchannel assembly. $(\mathrm{g})$ Photo of fabricated system.

Figure 5: (a) Graph showing the resonant frequency of the SAW sensor tracking the changes in E. coli biomass due to four different biofilm treatments initiated after 24 hours of biofilm growth. Only the BE demonstrates significant biomass reduction ( $80 \%$ reduction of total biomass). The error bar indicates the standard deviation of three repeats at selected time points. (b) Frequency shifts of the SAW sensor in four different $P$. aeruginosa biofilm treatments. The error bar indicates the standard deviation of three repeats at selected time points.

Figure 6: Representative fluorescence microscopy images obtained after different biofilm treatments. The green bacteria indicate live $E$. coli cells in the biofilm. Percentage values of the surface coverage of the biofilm in the images are calculated using the Image $J$ software: (a) control (38.1\%), (b) antibiotic (22.6\%), (c) electric field (41.1\%) and (d) BE (2.5\%). (e) Surface coverage. The BE treated E. coli biofilm shows significantly reduced live cells in the biofilm as compared to the traditional antibiotic therapy.

Figure 7: Representative fluorescence microscopy images after four different biofilm treatments. The green fluorescence indicates live $P$. aeruginosa bacterial cells in biofilm. Percentages of the surface coverage of the biofilms were obtained using the Image J software: (a) control (13.6\%), (b) antibiotic $(13.2 \%)$, (c) electric field (12.9\%) and (d) BE (1.7\%). (e) Surface coverage. P. aeruginosa biofilm treated by the BE shows significantly reduced live cells in biofilm compared to the traditional antibiotic therapy. 


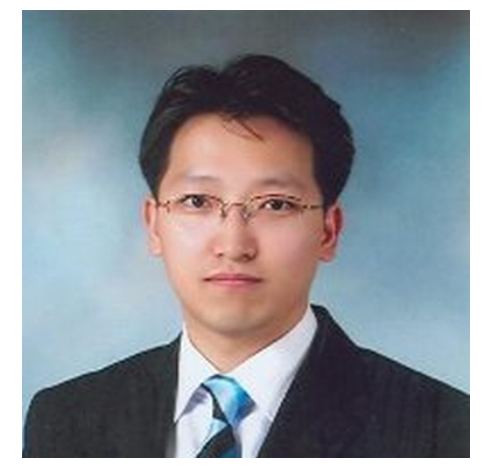

Young Wook Kim, Ph.D.

Young Wook Kim received B.S. in Electrical Engineering from Seoul National University in Feb. 2007. Prior to finish the B.S. in Seoul National University, he completed premedical courses from University of Ulsan College of Medicine in 2001, Korea. He received M.S. \& Ph.D. from the University of Maryland, College Park, USA, in 2011 and 2014 respectively, all from the Electrical Engineering department. In his Ph.D. work, he developed an integrated microsystem for bacterial biofilm infections real-time monitoring and treatment.

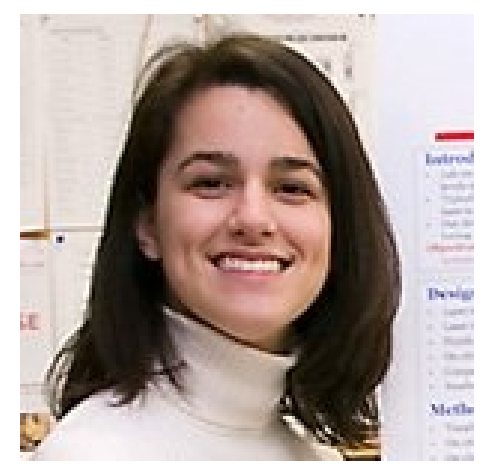

Mariana T. Meyer, Ph.D.

Mariana Meyer received B.S. in Mechanical Engineering from Stanford in June 2007, and received Ph.D. in Bioengineering at the University of Maryland, College Park, in January 2014. In her Ph.D. work, she worked on a microfluidic Lab-on-aChip system for biofilm characterization under the direction of Dr. Reza Ghodssi. 


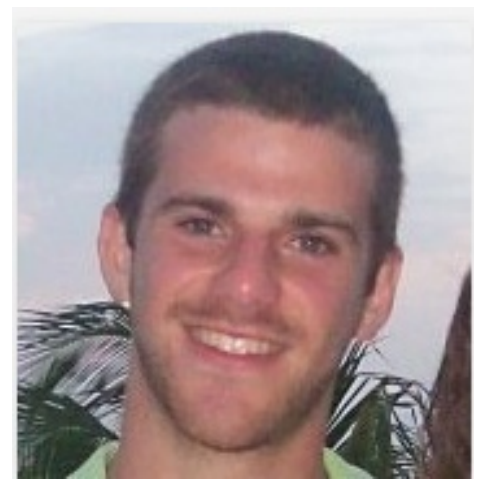

Andrew Berkovich

Andrew Berkovich received the B.Sc. and M.S. degrees in electrical engineering from the University of Maryland, College Park, MD, USA in 2011 and 2015, respectively. He is currently pursuing the Ph.D. degree at the University of Maryland, College Park, where his research focuses on biologically-inspired vision systems for autonomous aerial platforms. His research interests include computational sensing, mixed-signal very large scale integration (VLSI) systems, and neuromorphic sensory circuits and systems.

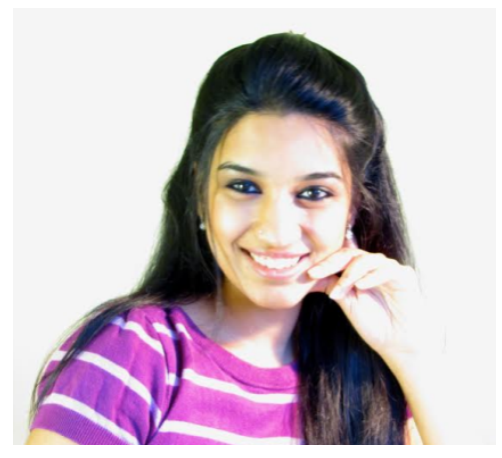

Sowmya Subramanian

Sowmya Subramanian completed her B.E in Electrical Engineering (EE) and M.Sc in Biological Sciences from the Birla Institute of Technology and Science (BITS), Pilani, India in 2007. After working for a couple of years in the industry, she joined the University of Maryland, College Park in 2009 to pursue her master's in EE during which she worked on using carbon nanotube field effect transistors (CNTFETs) to detect pathogenic E.coli in food samples. Currently, her PhD research focuses on real-time bacterial biofilm detection and treatment for in-vivo clinical applications using Lab-on-a-Chip technology. 


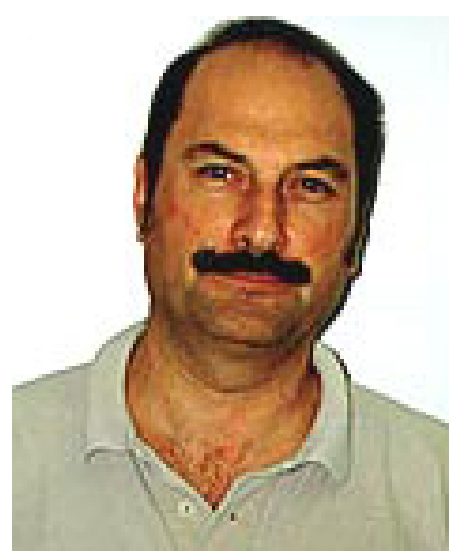

Agis A. Iliadis, Ph.D.

Professor Agis A. Iliadis is the Director of the Semiconductor Nanotechnology Research Laboratory and a member of the Maryland NanoCenter. He received his M.Sc. and Ph.D. degrees in Electrical Engineering, from the Department of Electrical Engineering and Electronics, University of Manchester Institute of Science and Technology (UMIST). His expertise is in the areas of nanotechnology, sensors, semiconductor devices/circuits, and CMOS IC technology. Current research focus is in novel sensors and nanotechnology compatible with CMOS IC technology for developing multi-functionality in CMOS ICs, smart sensor arrays, and nanodevices. His contributions are in the development of self-assembled nanostructures and nanocomposite copolymers on $\mathrm{Si}$ CMOS wafers, $\mathrm{ZnO}$ and SiC wide band-gap semiconductor devices and technology, gas sensors, heterojunction field effect transistors (HEMTs), SOI MOSFETs, light emitting devices in III-V semiconductors, light emission in $\mathrm{Si}$, ohmic contacts, thin film epitaxial growth, improved metal/semiconductor and oxide/semiconductor interfaces, and electromagnetic interference (EMI) in CMOS integrated circuits. 


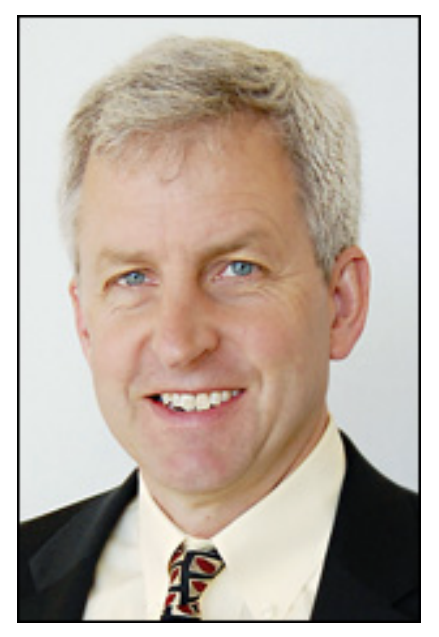

William E. Bentley, Ph.D.

William E. Bentley is the Robert E. Fischell Distinguished Professor of Engineering and founding Chair of the Fischell Department of Bioengineering. He is also appointed to the Department of Chemical and Biomolecular Engineering at the University of Maryland, College Park and the Institute for Bioscience and Biotechnology Research. At Maryland since 1989, Dr. Bentley has focused his research on the development of molecular tools that facilitate the expression of biologically active proteins, having authored over 270 related archival publications.

He is a fellow of AAAS, ACS, AIMBE, and the American Academy of Microbiology. He has served on advisory committees for the NIH, NSF, DOD, DOE, FDA, USDA, and several state agencies and has mentored more than $30 \mathrm{PhDs}$ and 15 postdocs, many now in leadership roles within industry (18), federal agencies (4) and academia (22). He co-founded a protein manufacturing company, Chesapeake PERL, based on insect larvae as mini bioreactors. 


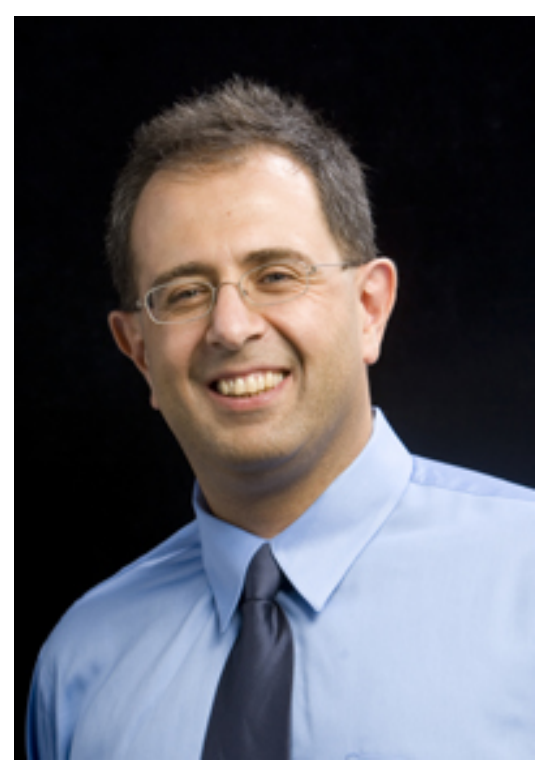

Reza Ghodssi, Ph.D.

Reza Ghodssi is the Herbert Rabin Distinguished Chair in Engineering, Director of the Institute for Systems Research (ISR) and Director of the MEMS Sensors and Actuators Lab (MSAL) in the Department of Electrical and Computer Engineering (ECE) and the Institute for Systems Research (ISR) at the University of Maryland (UMD). Dr. Ghodssi's research interests are in the design and development of microfabrication technologies and processes in micro/nano/bio devices and systems for chemical and biological sensing, small- scale energy conversion and harvesting with a strong emphasis toward health monitoring applications. Dr. Ghodssi is a Fellow of the IEEE, AVS, and ASME, a University of Maryland Distinguished Scholar-Teacher, has more than 125 journal publications and 290 refereed conference papers, and is the co-editor of the MEMS Materials and Processes Handbook published in 2011. Dr. Ghodssi is an associate editor for the Journal of Microelectromechanical Systems (JMEMS) and Biomedical Microdevices ( $\underline{B M M D})$. 
Figure 1

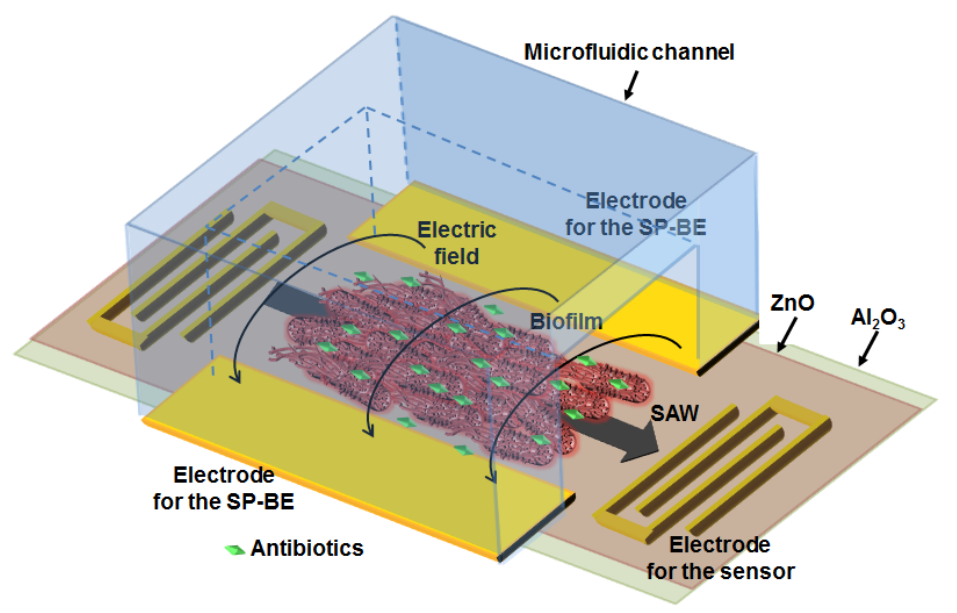

Figure 1: Schematic of the integrated microsystem of the SAW sensor and electrodes for induction of the bioelectric effect. A microfluidic chamber is placed over the system to introduce bacterial growth media and antibiotics. 
Figur e 2

(a)

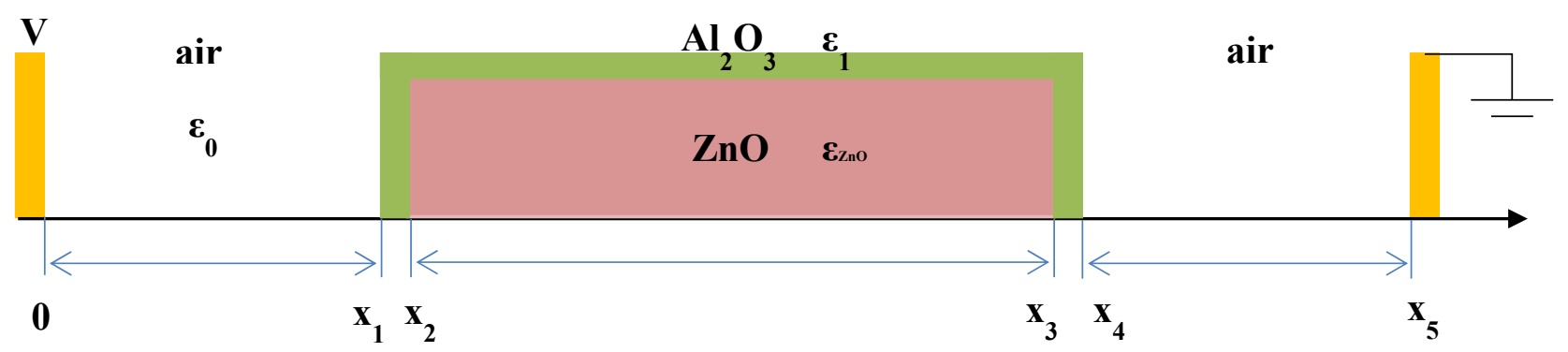

(b)

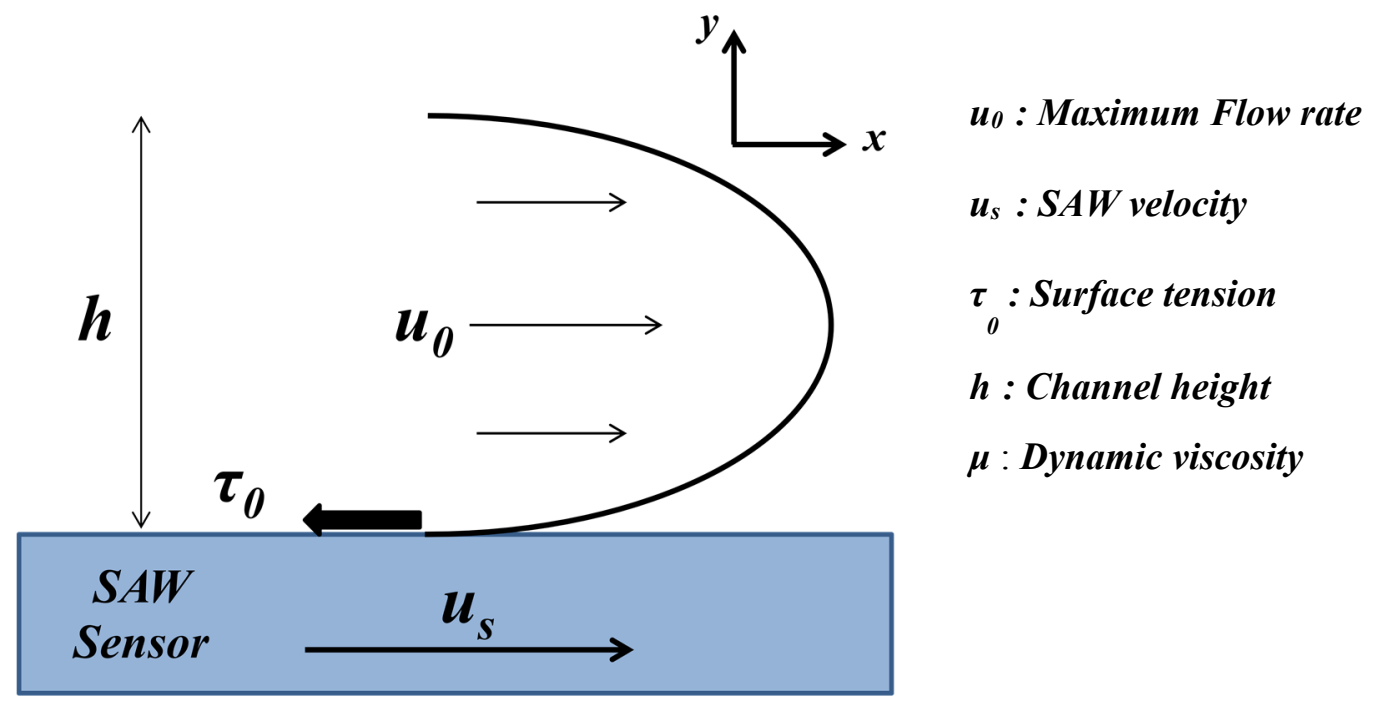

Figure 2: (a) Schematic of the device cross-section for electric field distribution analysis. The $\mathrm{ZnO}$ can affected by the voltage applied for the bioelectric effect during the biofilm treatment. The simulation was focused on the $\mathrm{ZnO}$ layer from $\mathrm{X}_{2}$ to $\mathrm{X}_{3}$ locations. $\varepsilon$ represents a dielectric constant of the each medium. (b) Schematic of the laminar flow on the SAW. The theoretical analysis was focused on the impact of the SAW speed changes due to the flow. 
Figure 3

(a)

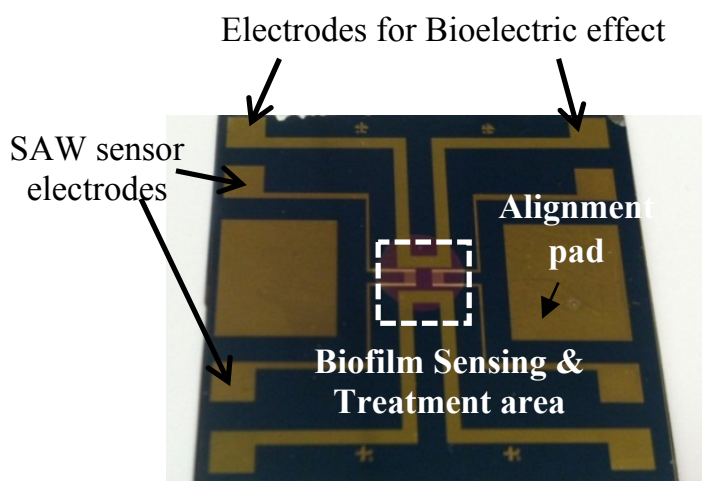

(b)

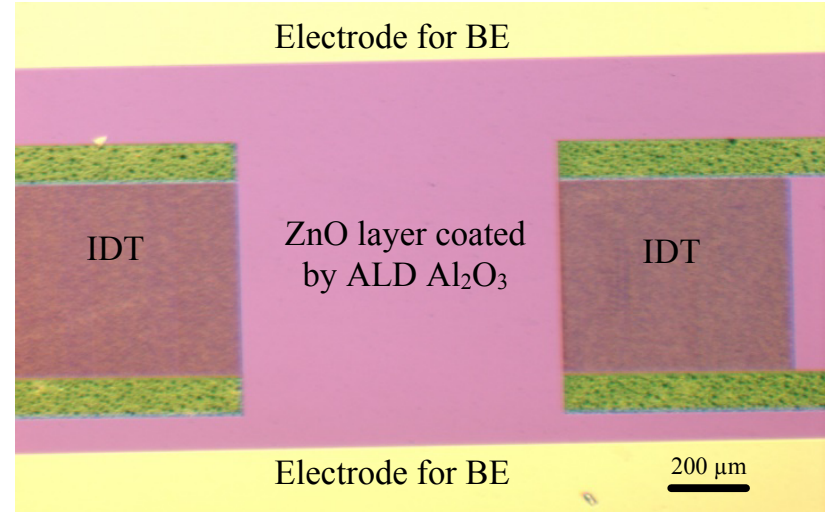

Figure 3: (a) Photo of the fabricated device. (b) Optical microscopy image of the integrated device. Biofilm is localized between the electrodes for the bioelectric effect (BE) via a microfluidic channel. Biofilm sensing is based on the total biomass between the two IDTs and treatment is applied when electric signal was applied to the electrodes for the $\mathrm{BE}$ as antibiotics are supplied through the microchannel. 
(a)

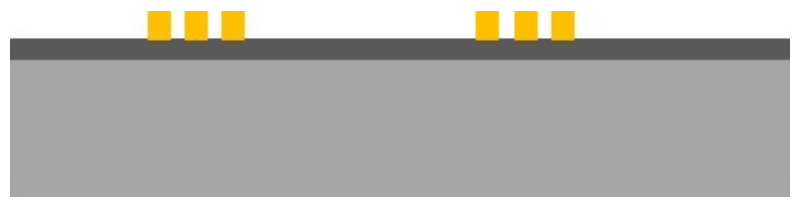

(c)

\section{뭄

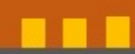

(e)

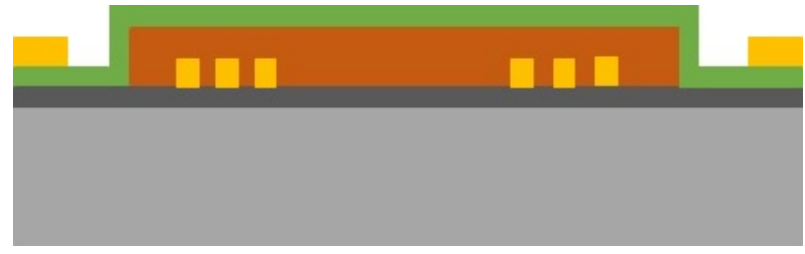

(f)

(g)

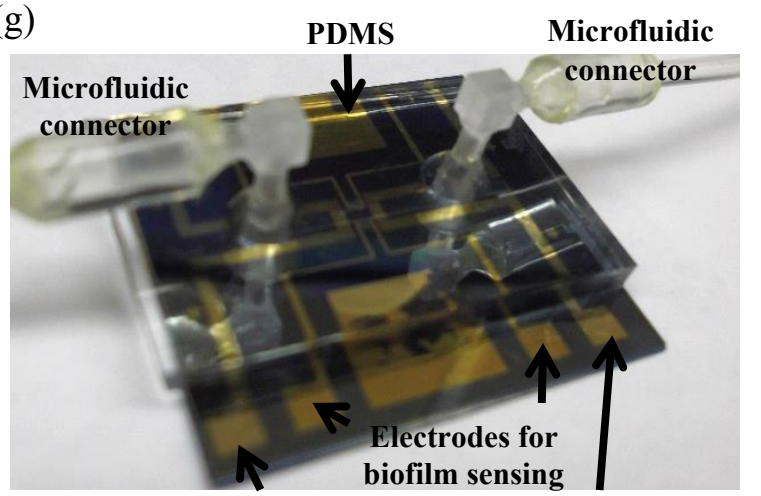

Electrodes for biofilm treatment

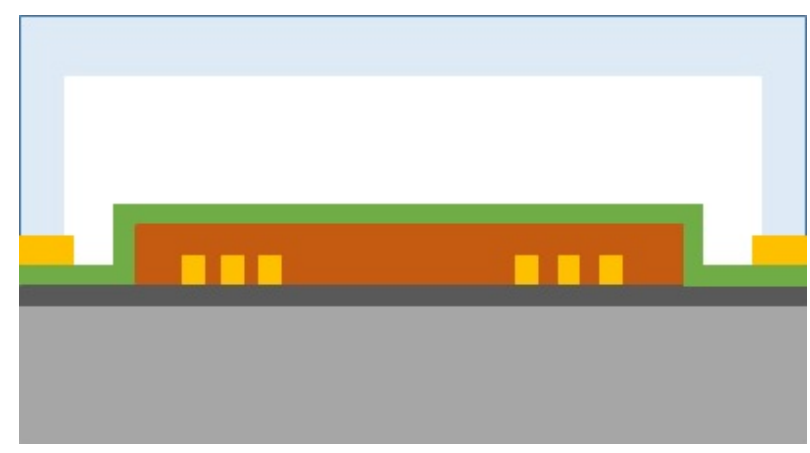

(b)

\section{ㅁㅁ \\ प्त}

(d)
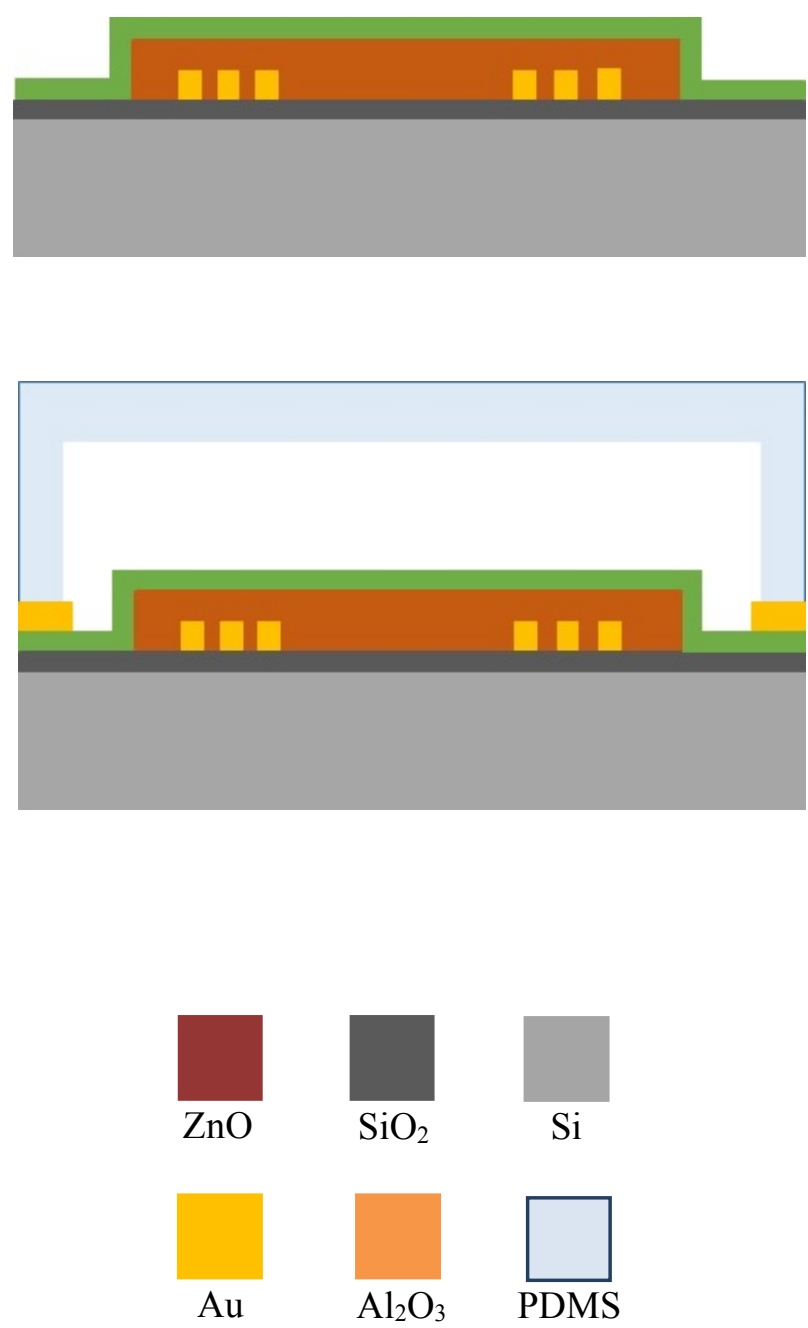

Figure 4: Overall schematic process flow. (a) $60 \mathrm{~nm} \mathrm{PECVD} \mathrm{SiO}_{2}$ deposition and interdigitated electrode patterning and deposition ( $\mathrm{Au}$ ) by electron beam evaporation, (b) $500 \mathrm{~nm} \mathrm{ZnO}$ deposition using pulsed laser deposition, (c) $\mathrm{ZnO}$ patterning using weak acid etchant (1:1:30 ratio of acetic acid: phosphoric acid: deionized water), (d) $100 \mathrm{~nm}$-thick atomic layer deposition of $\mathrm{Al}_{2} \mathrm{O}_{3}$ at $150{ }^{\circ} \mathrm{C}$, (e) electrode patterning and fabrication via $\mathrm{Au}$ evaporation $(\mathrm{Cr} / \mathrm{Au}, 15 \mathrm{~nm} / 200 \mathrm{~nm}$ ) and (f) PDMS microchannel assembly. (g) Photo of fabricated system. 
(a)

E. coli biofilm experiments

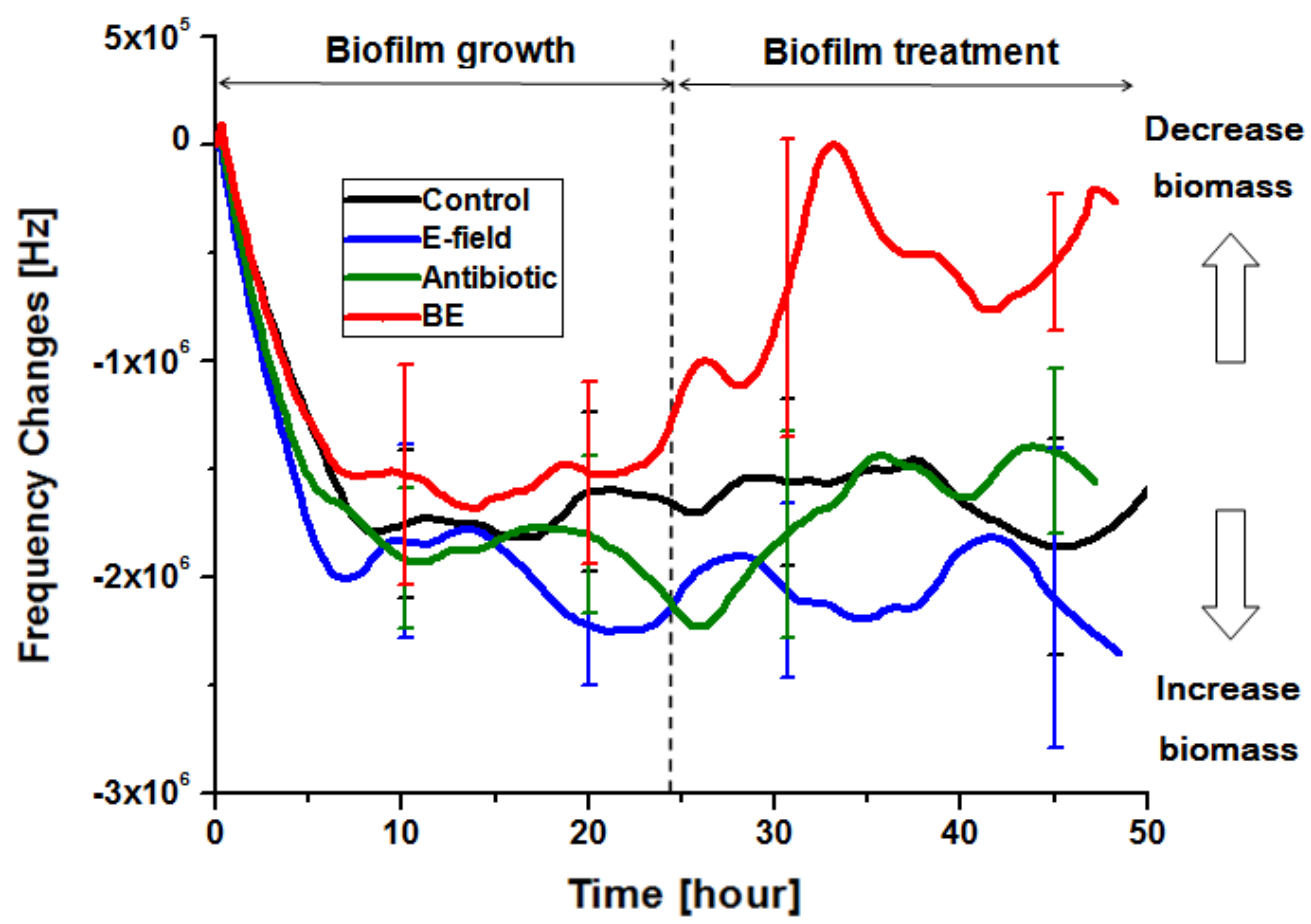

(b)

$P$. aeruginosa biofilm experiments

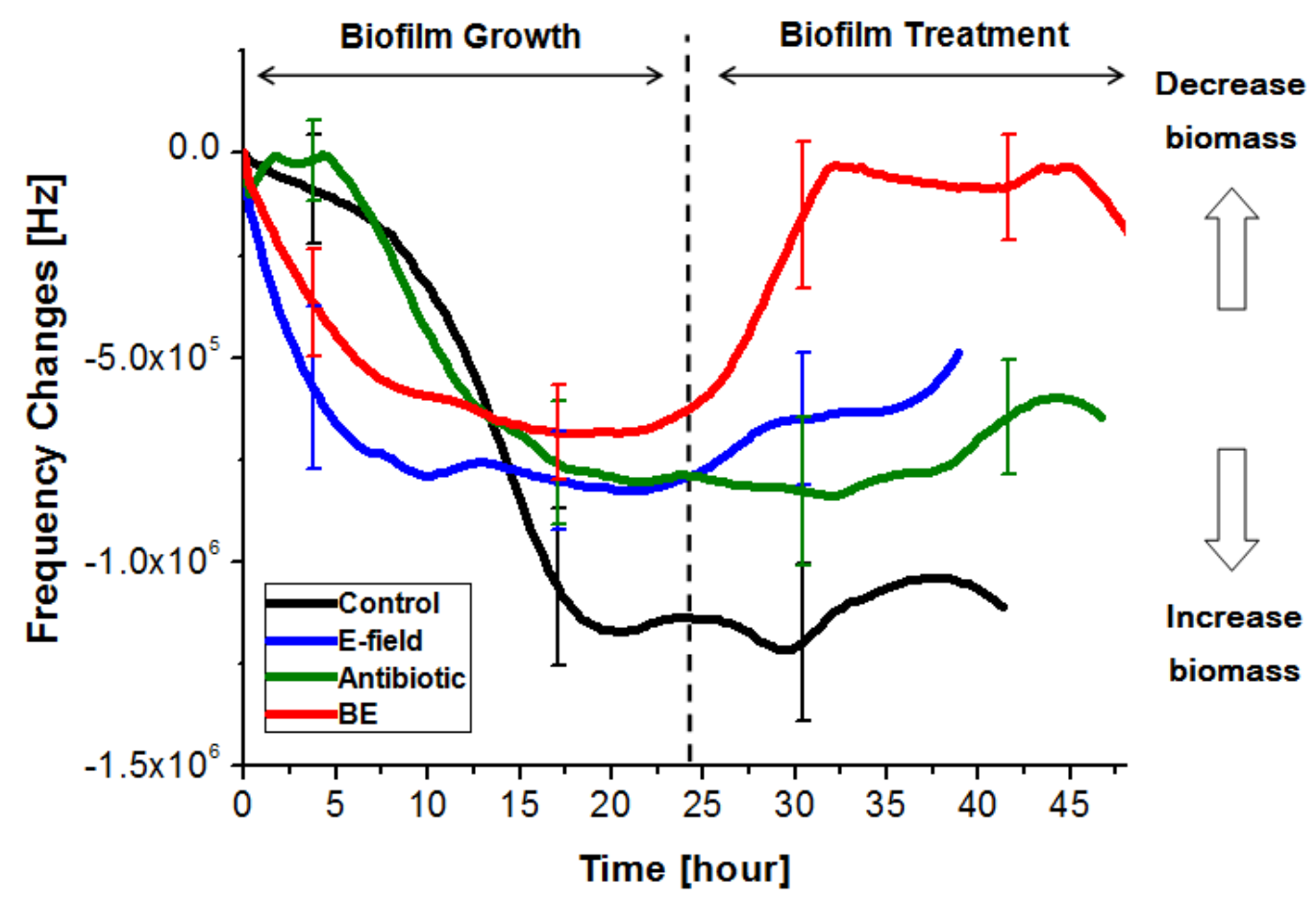


Figure 5: (a) Graph showing the resonant frequency of the SAW sensor tracking the changes in E. coli biomass due to four different biofilm treatments initiated after 24 hours of biofilm growth. Only the BE demonstrates significant biomass reduction ( $80 \%$ reduction of total biomass). The error bar indicates the standard deviation of three repeats at selected time points. (b) Frequency shifts of the SAW sensor in four different $P$. aeruginosa biofilm treatments. The error bar indicates the standard deviation of three repeats at selected time points. 


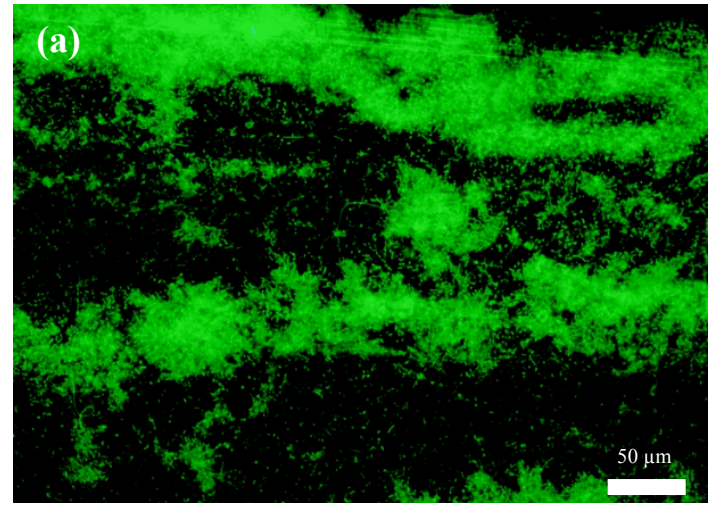

Control (no treatment)

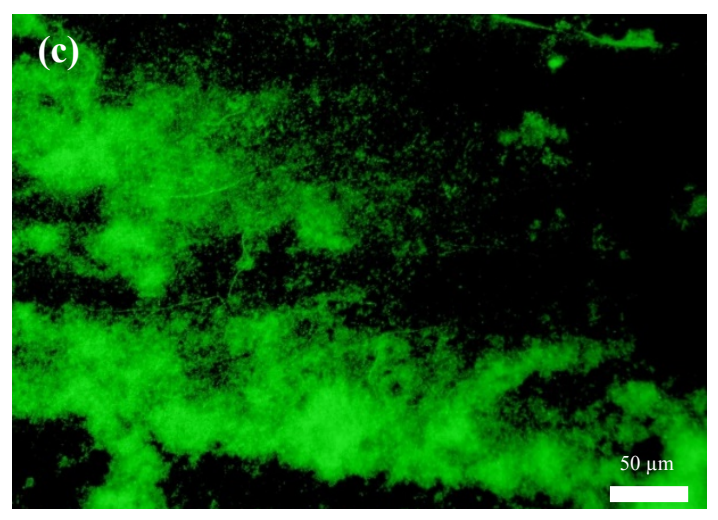

Electric field (AC + DC fields)

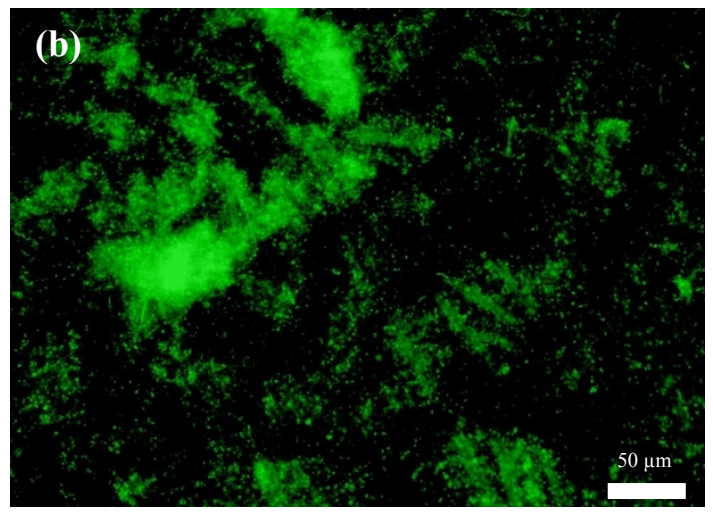

Antibiotic (Gentamicin $10 \mu \mathrm{g} / \mathrm{mL}$ )

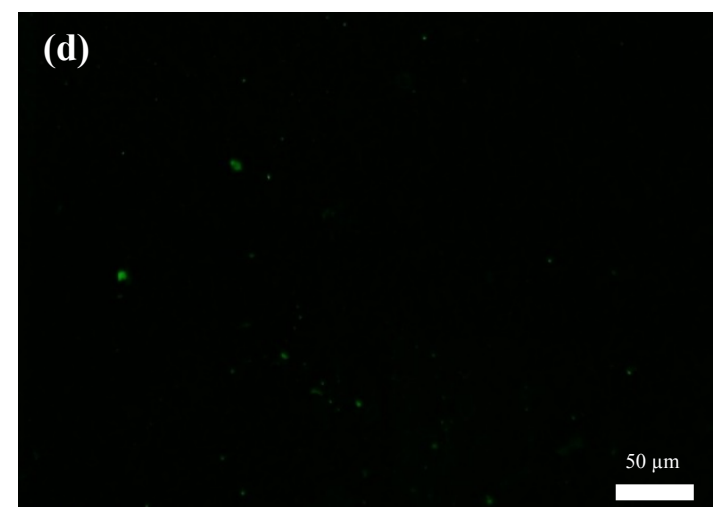

BE (Electric field + Gentamicin)

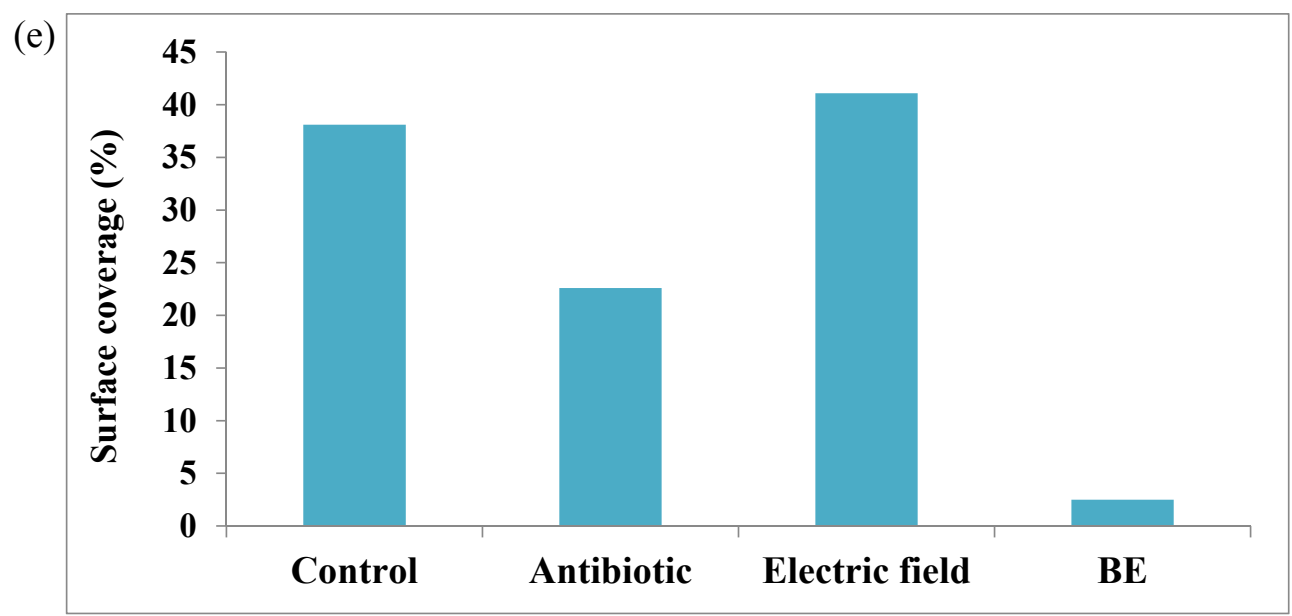

Figure 6: Representative fluorescence microscopy images obtained after different biofilm treatments. The green bacteria indicate live E. coli cells in the biofilm. Percentage values of the surface coverage of the biofilm in the images are calculated using the Image J software: (a) control $(38.1 \%)$, (b) antibiotic (22.6\%), (c) electric field (41.1\%) and (d) BE (2.5\%). (e) Surface coverage. The BE treated E. coli 
biofilm shows significantly reduced live cells in the biofilm as compared to the traditional antibiotic therapy.

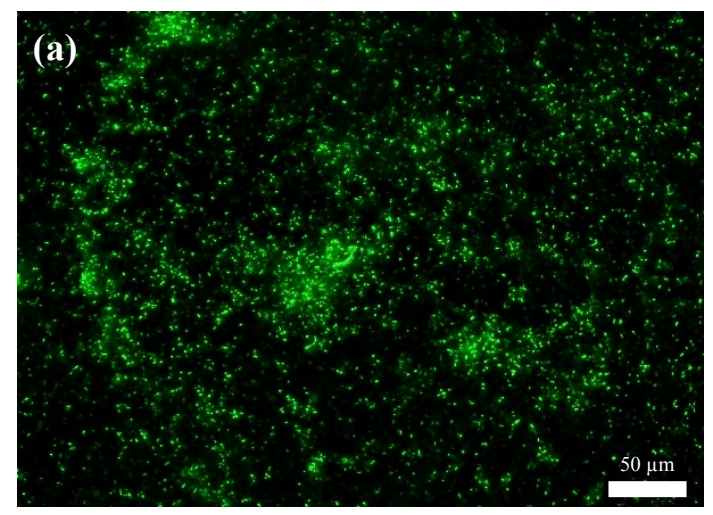

Control (no treatment)

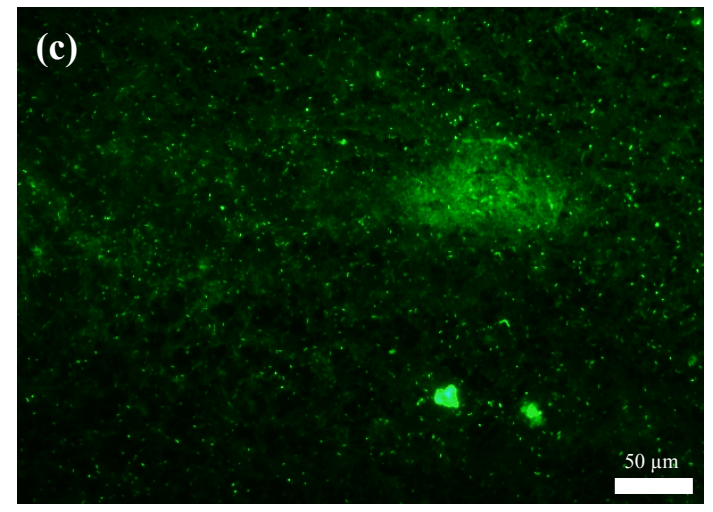

Electric field (AC+DC fields)

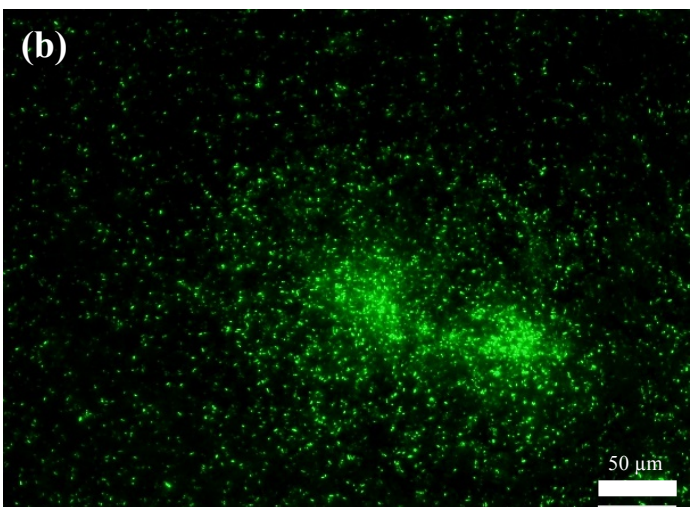

Antibiotic (Gentamicin $10 \mu \mathrm{g} / \mathrm{mL}$ )

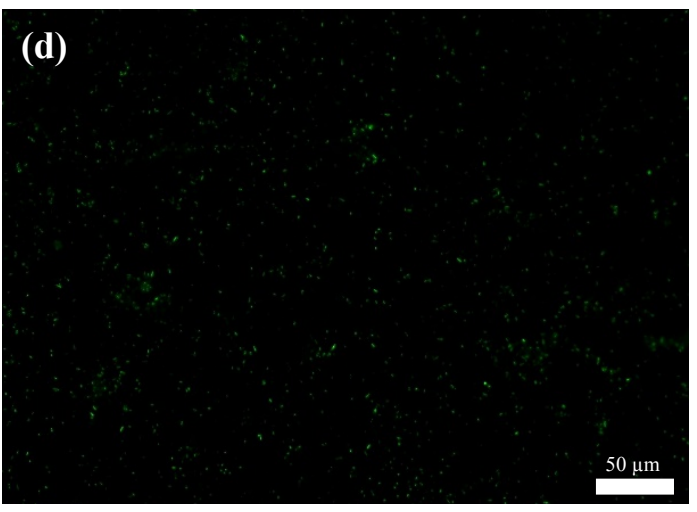

$\mathrm{BE}($ Electric field + Gentamicin)

(e)

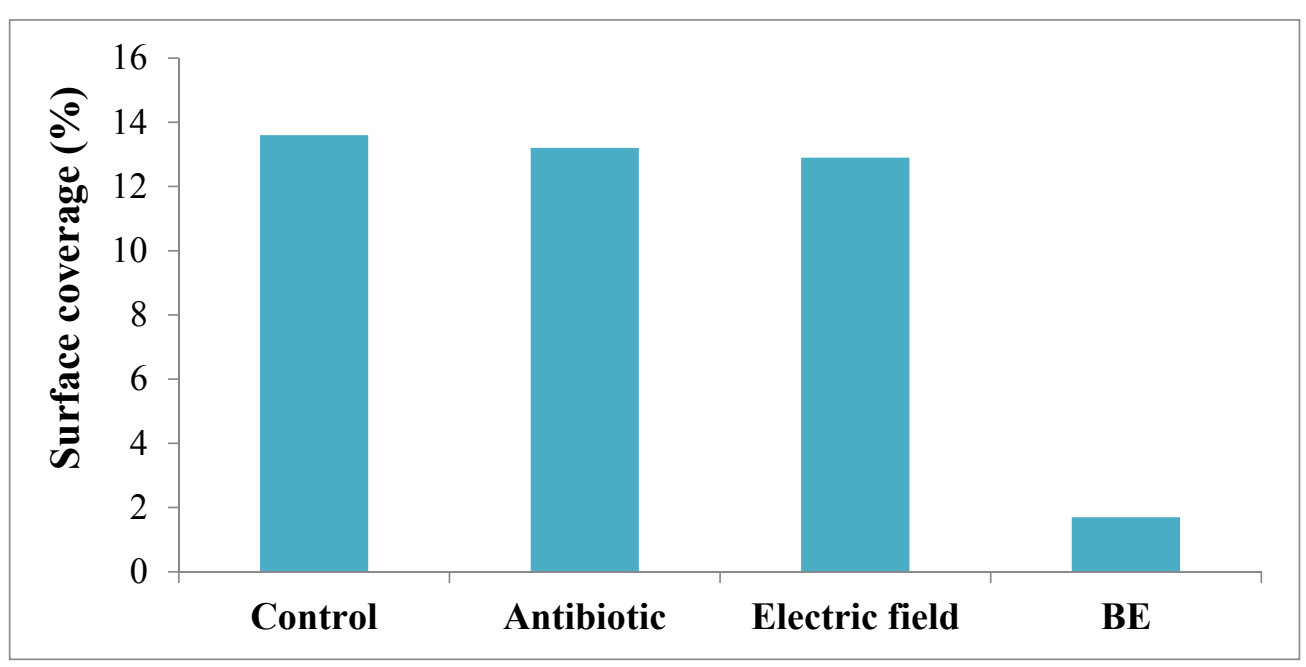


Figure 7: Representative fluorescence microscopy images after four different biofilm treatments. The green fluorescence indicates live $P$. aeruginosa bacterial cells in biofilm. Percentages of the surface coverage of the biofilms were obtained using the Image $J$ software: (a) control (13.6\%), (b) antibiotic (13.2\%), (c) electric field (12.9\%) and (d) BE (1.7\%). (e) Surface coverage. P. aeruginosa biofilm treated by the BE shows significantly reduced live cells in biofilm compared to the traditional antibiotic therapy. 
Table 1: Summary of the detection limit of the device

\begin{tabular}{|c|c|}
\hline ZnO Thickness & $\sim 500 \mathrm{~nm}$ \\
\hline $\mathbf{A l}_{\mathbf{2}} \mathbf{O}_{\mathbf{3}}$ Thickness & $110 \mathrm{~nm}$ \\
\hline $\boldsymbol{\Delta f}$ & $125 \mathrm{KHz}$ \\
\hline Detection Limit & $166 \mathrm{pg}$ \\
\hline
\end{tabular}

